\title{
Efficacy and Safety of Low- Dose Aspirin for Venous Ulcers: Randomized Clinical Trial
}

Keywords: Aspirin; Venous ulcers; VLUs

\section{Abstract}

Background: Venous leg ulcers are chronic relapsing wounds which impair quality of life and has significant health economic burden. Many venous leg ulcers take over 6 months to heal and a quarter will fail to heal completely. Current standard treatments include compression therapy, bandages and Pentoxifylline.

Few published trials in United Kingdom and Spain demonstrated that Aspirin may hasten healing of VLUs.

Methods: A prospective, randomized, double-blind, placebo-controlled trial was conducted to provide evidence regarding the efficacy and safety of Aspirin in addition to standard care. Patients aged I8 to 75 with at least one chronic venous leg ulcer were recruited. Both the treatment and placebo groups were instructed to take 2 tablets of $80 \mathrm{mg}$ Aspirin once daily after breakfast for a maximum of I2 weeks or less in case of complete ulcer healing. Eligible patients were stratified according to size ( $\leq 5 \mathrm{~cm} 2$ and $>5 \mathrm{~cm} 2$ ). The primary outcome measure was the proportion of patients with wound closure described as eschar formation over the entire surface. Safety outcomes were assessed in all participants.

Results: A total of 34 patients were enrolled in the study. In the aspirin group, $33.33 \%$ of patients had complete ulcer closure compared with $0 \%$ in the control group (RR 0.6667, 95\% CI 0.466I to 0.9535, P = 0.0264). Mean healed area of ulcer in the aspirin group was $36.19 \mathrm{~cm} 2$ compared to $21.39 \mathrm{~cm} 2$ in the control group. Healing time for ulcers in the aspirin group was at $6 \pm 2.19$ weeks.

Conclusion: This study demonstrated that Aspirin 160mg has a favorable effect and can be given as an adjunct to standard wound care and compression in the management of venous ulcers. Proper selection of patients will prevent adverse effects and improve healing rate and time.

\section{Introduction}

Venous leg ulcers are a type of chronic wound affecting up to $1 \%$ of adults in developed countries at some point during their lives [1]. It accounts for 80 percent of lower extremity ulcerations [2]. The venous leg ulcer is an age related disease in the elderly population, especially women. The primary risk factors are older age, obesity, previous leg injuries, deep venous thrombosis, and phlebitis [3].

Dormandy, in reviewing the pathophysiology of venous leg ulcers, stated that the currently favored hypothesis for the link between the increased venous pressure of chronic venous insufficiency and venous ulcer is based on the intermittent inappropriate activation of white blood cells [4]. The damage initiated by the oxidative burst of the leukocytes leads to endothelial dysfunction, interstitial edema, microthrombi, and long-term microcirculatory damage, including decreased capillary density. The net result is impairment of the potential for healing and hence ulcer formation [5].

Whereas the venous leg ulcer is usually originated by external trauma, the course is often chronic and/or relapsing [3]. Many venous leg ulcers take over 6 months to heal; one large study demonstrated

\section{Journal of}

\section{Clinical \& Investigative} Dermatology

\author{
Sanchez-Dipasupil E*, Gulmatico-Flores Z and Lopez- \\ Villafuerte $\mathbf{L}$ \\ Department of Dermatology, Jose R. Reyes Memorial Medical Center, \\ Philippines
}

\begin{abstract}
*Address for Correspondence
Sanchez-Dipasupil E, Department of Dermatology, Jose R. Reyes Memorial Medical Center, Philippines, Phone: +639178777978; E-mail: edessahmd@gmail.com

Submission: 16 November, 2019

Accepted: 20 December, 2019

Published: 23 December, 2019

Copyright: ๑ 2019 Sanchez-Dipasupil E, et al. This is an open access article distributed under the Creative Commons Attribution License, which permits unrestricted use, distribution, and reproduction in any medium, provided the original work is properly cited.
\end{abstract}

a median time to ulcer healing of 99 days with 2-layer compression therapy. In addition, more than a quarter fail to heal completely and the 12-month recurrence rate of healed venous leg ulcers may be up to $28 \%$. Patients with longstanding, large ulcers, or who have a prior history of ulceration, are particularly resistant to healing [6].

Therearevarious treatmentoptionsforvenousulcers. Theseinclude conservative management, mechanical treatment, medications, and surgical options. Evidence rating a key recommendation includes compression therapy, bandages and Pentoxifylline [2]. Compression assists by reducing venous hypertension, enhancing venous return and reducing peripheral edema. However, studies show that it only has moderate effects on healing, with up to $50 \%$ of venous leg ulcers unhealed after two years of compression. Non-adherence may be the principal cause of these poor results, but presence of inflammation in people with chronic venous insufficiency may be another factor, so a treatment that suppresses inflammation and reduces the frequency of ulcer recurrence would be an invaluable intervention to complement compression treatments [7].

Aspirin is a cyclooxygenase inhibitor that irreversibly reduces prostaglandin-2 and thromboxane A2, which are involved in inflammation and platelet aggregation. It is inexpensive, widely used and readily available. The mechanism by which aspirin may hasten healing of VLUs is unclear but may be associated with a reduction of inflammation, or its effect on the microvascular circulation, including platelet activation. In one study investigating the haemostatic effects of aspirin in patients with VLU, the investigators demonstrated that participants were found to have increased levels of fibrinogen and shortened coagulation rate, when compared to age-matched and sexmatched controls and that treatment with aspirin caused prolongation of the coagulation rate, which increased the rate of ulcer healing [6].

Venous leg ulcers impair Quality of Life (QoL); they are open wounds, which can be large, are often painful, frequently become infected and leak exudate. It has significant health economic burden. There is, therefore, an unmet need for a more cost-effective and clinically effective treatment for VLUs. 
Citation: Sanchez-Dipasupil E, Gulmatico-Flores Z, Lopez-Villafuerte L. Efficacy and Safety of Low-Dose Aspirin for Venous Ulcers: Randomized Clinical Trial. J Clin Investigat Dermatol. 2019;7(2): 4

ISSN: 2373-1044

Table 1: Population characteristics

\begin{tabular}{|c|c|c|}
\hline Subjects $(\mathbf{n = 3 4 )}$ & Aspirin $(\mathbf{n = 1 7 )}$ & Placebo $(\mathbf{n = 1 7})$ \\
\hline Age $($ mean \pm SD) & $53.71+/-10.41$ & $52.59+/-11.01$ \\
\hline Sex $(\mathrm{M}: \mathrm{F})$ & $6: 11$ & $7: 10$ \\
\hline Ulcer size $\left(\mathrm{cm}^{2}\right)$ & $2.51+/-1.91$ & $2.18+/-0.91$ \\
\hline Mean duration of ulcer & $21.88+/-14.79$ & 0.3001 \\
\hline
\end{tabular}

Table 2: Total proportion of patients with complete healing and time to healing in both groups.

\begin{tabular}{|r|r|}
\hline & Aspirin \\
\hline Total proportion healed,n(\%) & $5 / 10(33.33 . \%)$ \\
\hline Time to healing (weeks), mean \pm SD & $0 / 14(0)$ \\
\hline NA & $6 \pm 2.19$ \\
\hline
\end{tabular}

Table 3: Comparison of ulcer surface area $\left(\mathrm{cm}^{2}\right)$ at baseline and at 12 weeks in both groups.

\begin{tabular}{|c|c|c|c|}
\hline & Baseline $\left(\mathbf{c m}^{2}\right)$ & Mean at $\mathbf{1 2}$ weeks $\left.\mathbf{( c m}^{2}\right)$ & Percentage of surface area healed \\
\hline Aspirin & $2.51 \pm 1.85$ & $0.43 \pm 0.22$ & $87.53 \%$ \\
\hline Placebo & $2.18 \pm 0.088$ & $1.12 \pm 0.40$ & $52.91 \%$ \\
\hline
\end{tabular}

\section{Methodology}

\section{Patients and research design}

This is a prospective, randomized, double-blind, placebocontrolled trial to provide evidence regarding the efficacy and safety of aspirin, at a dose of $160 \mathrm{mg}$ once daily, in addition to standard care in patients with chronic venous leg ulcers conducted at the Jose R. Reyes Memorial Medical Center Dermatology Outpatient Department.

Patients recruited included male and female patients aged 18 to 75 with at least one chronic venous leg ulcer confirmed by duplex scan to be venous in origin. Patients included should be willing to have elastic compression therapy. The ulcers had to be open for at least 6 weeks.

The exclusion criteria included those who are unable or unwilling to provide consent, patients with diabetes mellitus and/or peripheral arterial occlusive disease, those with leg ulcers of non-venous etiology, on regular concomitant aspirin, with previous intolerance or contraindication to aspirin use, taking prohibited medications such as, probenecid. Oral anticoagulants including coumarins and phenindione, dabigatran, heparin, clopidogrel, dipyridamole, sulfinpyrazone and iloprost, with history of peptic ulcer / dyspepsia, with known lactose intolerance, pregnant or lactating women, patients

with infection of deeper skin structures or with intensive involvement requiring systemic antibiotics, those who have been given oral or topical antibiotics within 2 weeks and/or other reasons that excludes them from participating within this trial made by the investigator's clinical judgment.

Approval from the Institutional Review Board of the hospital was obtained prior to commencement of the trial. Eligible patients were asked to sign a written informed consent prior to their inclusion to the study. All patients were fully informed about the nature of the research study and the chances of being randomized to either the trial drug (aspirin) or placebo. Eligible patients were stratified according to size $\left(\leq 5 \mathrm{~cm}^{2}\right.$ and $\left.>5 \mathrm{~cm}^{2}\right)$. This size stratification was used because venous ulcers $>5 \mathrm{~cm}^{2}$ have been shown to have slower closure rates than smaller wounds.

\section{Materials}

The aspirin $(80 \mathrm{mg})$ and placebo tablets of the same size, color and shape were placed in identical bottles. The bottles were labelled as $\mathrm{A}$ or B by a co-investigator.

\section{Randomization, treatment allocation, and blinding}

A computer-generated random sequence was used for allocation of recruited subjects. Subjects and investigator were blinded until after the completion of the study.

\section{Study intervention}

All participants underwent standardized, local management of ulcers which includes wound cleansing with saline solution, saline presses twice daily, compression therapy and leg elevation.

Patients under the treatment and placebo groups were instructed to take 2 tablets of given medication once daily after breakfast for a maximum of 12 weeks or less in case of complete ulcer healing. All participants were advised to avoid consumption of acidic foods such as alcoholic beverages and coffee.

Patients were also given a special instruction to immediately discontinue the medication and inform the investigator if they experience signs of gastric irritation.

\section{Clinical assessment}

All patients were evaluated by the same dermatologist at baseline and every 2 weeks until closure or a maximum of 12 weeks. Digital photographs and ulcer surface area were obtained every 2 weeks.

The primary outcome measure of the study was the proportion of patients with complete healing in each group described as eschar formation over the entire wound surface. Secondary outcome parameters included time to complete healing, percentage of surface area healed and the incidence of adverse events. 


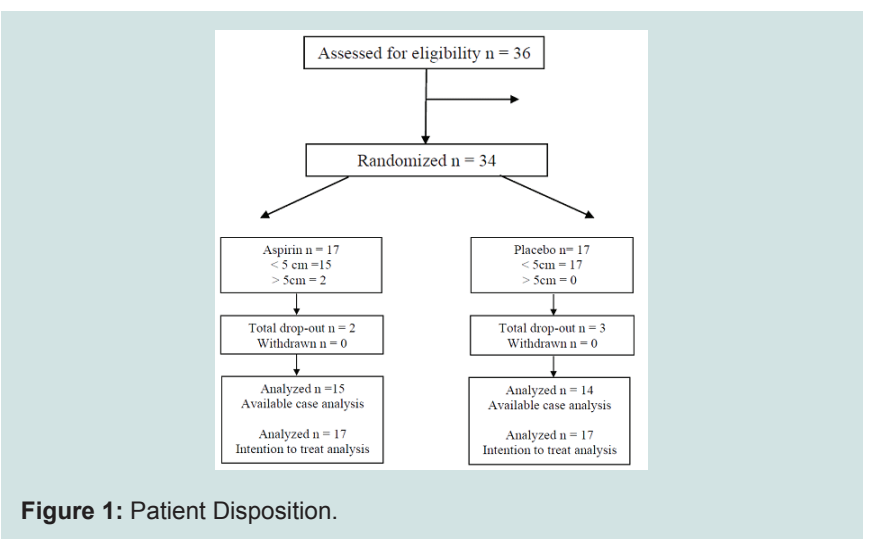

Figure 1: Patient Disposition.

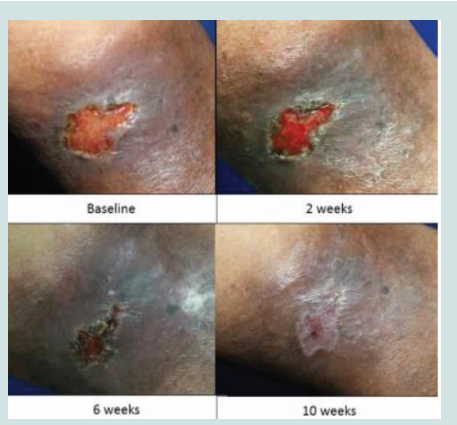

Figure 2: Wound healing of treatment group at baseline, 2 weeks, 6 weeks and complete closure at 10 weeks.

The surface area of the ulcer was measured using manual planimetry. Number of grids found within the traced circumference over an acetate film was counted and multiplied by the area in square centimeters. All partial grids of more than 50\% were included. Baseline CBC, creatinine, SGPT, SGOT, bleeding time, clotting time and urinalysis were requested.

\section{Stopping guidelines}

All patients who develop unacceptable treatment toxicity which, in the investigator's opinion, is attributable to the intervention were withdrawn from the study but follow-up was continued to enable an intention-to-treat analysis. The side effects associated with aspirin include, but are not limited to, gastrointestinal hemorrhage and gastrointestinal disturbance including dyspepsia, and ulceration. Those who had worsening of skin lesions were withdrawn and treated appropriately. Those who did not comply with the single daily dosing of the tablets, or those who used other medications were also withdrawn.

Dropouts were defined as those who did not follow up within 2 weeks and whose outcome were unknown by the end of the study period.

\section{Results}

Thirty-six patients were evaluated for eligibility between January 2017 and September 2017. Two patients diagnosed with diabetes mellitus were excluded. A total of 34 patients were enrolled in the study, with 17 patients assigned to each arm. Aspirin group had 2 drop outs owing to nonattendance at scheduled visits (Figure 1).
Majority of the subjects were females (61\%) with $64.71 \%(11 / 17)$ from the treatment group and $58.82 \%(10 / 17)$ from the placebo group. Mean age for both groups was 53.15, 53.71 years for the treatment group while 52.59 years for the control group. The mean duration of venous leg ulcer for the treatment group was 22 months and 24 months for the control group. Mean size of the ulcer on baseline was $2.35 \mathrm{~cm}^{2}$, measuring $2.51 \mathrm{~cm}^{2}$ for the treatment group and 2.18 $\mathrm{cm}^{2}$ for the placebo group. The baseline characteristics of the study population are summarized in (Table 1). T-test and chi-square tests were done. No statistically significant differences were noted between the two groups based on age, sex, duration of venous disease and baseline ulcer surface area.

\section{Clinical Effects}

In the aspirin group, $33.33 \%$ of patients had complete ulcer closure compared with $0 \%$ in the control group (RR 0.6667, 95\% CI 0.4661 to $0.9535, \mathrm{P}=0.0264$ ) (Table 2). All of these ulcers measured $\leq 5 \mathrm{~cm}^{2}$. There were only two subjects with ulcers $\geq 5 \mathrm{~cm}^{2}$ enrolled under the treatment group and none from the control group. Neither of these showed complete healing. Mean healed area of

ulcer in the aspirin group was $36.19 \mathrm{~cm}^{2}$ compared to $21.39 \mathrm{~cm}^{2}$ in the control group. Healing time for ulcers in the aspirin group were at $6 \pm 2.19$ weeks.

T-test was used to determine if there was a difference in the percentage of ulcer surface area after treatment between the treatment and control groups. With a p value of 0.0064 , the ulcer size of the treatment group significantly decreased in comparison with the placebo.

Relative risk reduction computation revealed that aspirin, in addition to standard wound care and compression, will improve venous ulcers $33 \%$ more. ARR was 0.33 , favouring aspirin with $33 \%$ chance of healing. NNT analysis revealed that three patients were
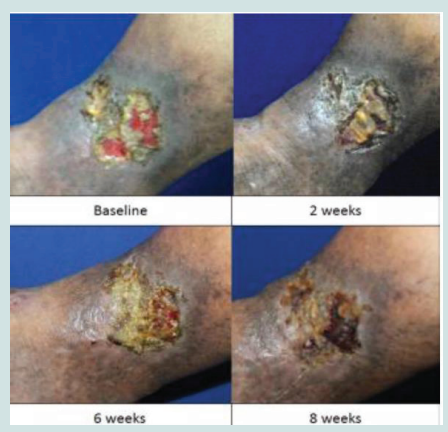

Figure 3: Wound healing of treatment group at baseline, 2 weeks, 6 weeks and 8 weeks.

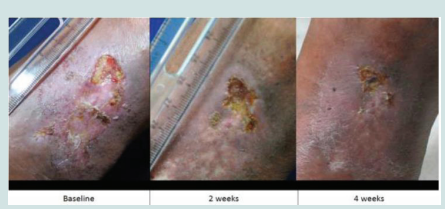

Figure 4: Wound healing of treatment group at baseline, 2 weeks and 4 weeks. 
Citation: Sanchez-Dipasupil E, Gulmatico-Flores Z, Lopez-Villafuerte L. Efficacy and Safety of Low-Dose Aspirin for Venous Ulcers: Randomized Clinical Trial. J Clin Investigat Dermatol. 2019;7(2): 4

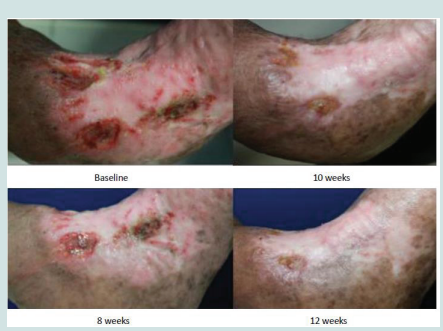

Figure 5: Wound healing of treatment group at baseline, 8 weeks, 10 weeks and 12 weeks.

required to be treated with aspirin to demonstrate complete ulcer closure. No adverse effects were noted in both groups (Figure 2-5).

\section{Discussion}

This study demonstrated that Aspirin has a favorable effect in the management of venous ulcers when used with compression stockings based on the higher proportion of healed ulcers, faster healing time and higher percentage of healed surface area. There were no adverse effects reported.

It was shown that administration of low dose aspirin is $33 \%$ more beneficial than the standard care alone and increases the chances of healing also by $33 \%$. Time to healing was faster, with 6 weeks compared to 99 days median time of ulcer healing with 2-layer compression therapy [6]. Although the difference was significant, it is important to note that those patients who demonstrated complete healing had ulcers less than $5 \mathrm{~cm}^{2}$ and duration of 12 months.

In both groups, better healing outcomes were seen in patients who had ulcers of shorter duration and smaller surface area. These results are in accordance with studies that investigated the associations of different risk factors and complete ulcer healing, which determined that longer ulcer duration and larger baseline surface areas were poor prognosticators of healing, and that age and sex did not seem to affect the outcome.

Result of this study was comparable with the two published clinical trials done in United Kingdom and Spain. Limitation of this study is the small sample size, short observation period, and failure to investigate recurrence.

There were no adverse effects documented in this study. However, complete evaluation of patients prior to administration of Aspirin is prudent. Lanas et al. did a literature review to construct risk-ratio estimations and determined incidences of cardiovascular and upper gastrointestinal complications according to the presence of different risk factors. Based on the results reported by Hernandez-Diaz and Garcia-Rodriguez, the pooled relative risk of upper GI bleeding was
2.0 for LDA, considered as doses $\leq 325 \mathrm{mg} /$ day. Major risk factors for the development of upper GI bleeding are: age, male gender, history of peptic ulcer and concomitant use of NSAIDs, anticoagulants, or clopidogrel. They presented clinical variables with corresponding relative risk ratio estimators. Of note, age $15-49$ has a RR of 1.0 and co-therapy with proton pump inhibitor has an RR of 0.4, decreasing the risk of bleeding by $60 \%$ [11- 13$]$.

\section{Conclusion}

Aspirin $160 \mathrm{mg}$ daily can be given as an adjunct to standard wound care and compression in the management of venous ulcers. Proper selection of patients will prevent adverse effects and improve healing rate and time.

\section{References}

1. O'Meara S, Al-Kurdi D, Ologun Y, Ovington LG, Martyn-St James M, et al. (2013) Antibiotics and antiseptics for venous leg ulcers. Cochrane Database Syst Rev 23: CD003557.

2. Lauren Collins, Seraj S, Thomas Jefferson University Hospital, Philadelphia, Pennslyvania (2010) Diagnosis and Treatment of Venous Ulcers. Am Fam Physician 8: 989-996.

3. Sarkar PK, Ballantyne S (2000) Management of ulcers. Postgrad Med J 76 674-682.

4. Dormandy A (1997) Pathophysiology of venous leg ulceration. Int J Microcirc Clin Exp 17: 2-5

5. Olivencia JA (2001) Pathophysiology of Venous Ulcers: Surgical Implications, Review and Update. Dermatol Surg 25: 880-885.

6. Tilbrook H, Forsythe RO, Rolfe D, Clark L, Bland M, et al. (2015) Aspirin for Venous Ulcers: Randomised Trial (AVURT): study protocol for a randomised controlled trial. Trials 16: 513 .

7. de Oliveira Carvalho PE, Magolbo NG, De Aquino RF, Weller CD (2016) Oral aspirin for treating venous leg ulcers. Cochrane Database Syst Rev 2: CD009432.

8. Scottish Intercollegiate Guidelines Network (2010) Management of chronic venous leg ulcers. SIGN 120.

9. de Oliveira Carvalho PE, Magolbo NG, De Aquino RF, Weller CD (2016) Oral aspirin for treating venous leg ulcers. Cochrane Database Syst Rev 2: CD009432.

10. Dalen J (2006) Aspirin to prevent heart attack and stroke: what's the right dose? Am J Med 119: 198-202

11. Lanas, Polo-Tomás M, Casado-Arroyo R (2013) The Aspirin Cardiovascular/ Gastrointestinal Risk Calculator: A Tool to Aid Clinicians in Practice. Aliment Pharmacol Ther 37: 738-748.

12. Salome GM, Blanes L, Ferreira LM (2014) The Impact of Skin Grafting on the Quality of Life and Self-Esteem of Patients with Venous Leg Ulcers. World J Surg 38: 233-240.

13. Salome GM, Ferreira LM (2017) Impact of non-adherent Ibuprofen foam dressing in the lives of patients with venous ulcers. Rev Col Bras Cir 44: 116-124. 